eseconomía, vol. XV, núm. 52, primer semestre de 2020, pp. 47-68

\title{
Variables económicas y deterioro de la calidad de la cartera de hipotecas bursatilizadas en México
}

Margarita María Mosso Martínez *

Francisco López-Herrera**

(Recibido: abril 2019/Aceptado: septiembre 2019)

\section{Resumen}

Este artículo se ocupa del análisis de la relación entre el deterioro de la calidad de la cartera de hipotecas bursatilizadas en México y variables económicas y financieras relevantes. Mediante un modelo autorregresivo de rezagos distribuidos (ARDL), se recolectó evidencia sobre la importancia de ciertas variables para explicar ese deterioro. La selección de variables se basó en la revisión de la literatura nacional e internacional, relacionada tanto con el riesgo sistemático como con el incumplimiento de los préstamos hipotecarios. El conocimiento de las variables causales de dicho deterioro puede ser útil para los emisores de valores respaldados por hipotecas, ya que podría ayudarlos a comprender mejor el riesgo que enfrentan esos valores. Naturalmente, este conocimiento también se puede utilizar para guiar la toma de decisiones de los inversionistas, así como de los administradores de portafolios y riesgos.

Palabras clave: calidad de carteras hipotecarias, cartera de hipotecas bursatilizadas, riesgo sistemático, riesgo macroeconómico.

Clasificación JEL: E44, F65, G10, G12, G21, N26.

* Profesor-investigador en la Universidad Anáhuac México

* Profesor-investigador en la Universidad Nacional Autónoma de México, División de Investigación, Facultad de Contaduría y Administración. 


\title{
Economic variables and spoilage of the quality portfolio of securitized mortgages in Mexico
}

\begin{abstract}
This paper deals with the analysis of the relationship between the spoilage of the quality of the portfolio of securitized mortgages in Mexico and relevant economic and financial variables. Through an autoregressive distributed lags (ARDL) model, evidence was collected on the importance of certain variables to explain that spoilage. The selection of variables was based on the review of national and international literature, related to both systematic risk and noncompliance with mortgage loans. Knowledge of the causal variables of such spoilage can be useful for issuers of mortgage-backed securities, as it could help them better understand the risk faced by those securities. Naturally, this knowledge can also be used to guide the decision making of investors, as well as portfolio and risk managers.
\end{abstract}

Keywords: Mortgage portfolio quality, securitized mortgage portfolio, systematic risk, macroeconomic risk.

JEL Classification: E44, F65, G10, G12, G21, N26.

\section{Introducción}

Al inicio del presente siglo se presentaron periodos de inestabilidad sin precedentes en los precios de las viviendas y en las condiciones del mercado inmobiliario, tanto en Estados Unidos como en otros países. En los últimos años, el financiamiento de la vivienda ha presentado una transformación cuya influencia incide en la incertidumbre de la deuda hipotecaria y de la actividad económica. Después del auge del crédito de vivienda durante mediados de la primera década del siglo XXI, las pérdidas de los prestamistas hipotecarios contribuyeron al desarrollo de una de las crisis económicas más importantes en la economía global. De acuerdo con Bullard et al. (2009), la crisis subprime tuvo entre 2007 y 2009 fuertes efectos financieros y económicos, originando a escala mundial el contexto conocido como la Gran Recesión, que se extendió hasta 2012 y puso en graves problemas financieros y económicos a los diversos países. 
Suárez (2006) señala que durante la década de 1970 los bancos de Estados Unidos especializados en hipotecas agruparon hipotecas residenciales y emitieron en los mercados bursátiles valores respaldados por dichos activos, encontrando así las instituciones financieras y los fondos de inversión inmobiliaria transnacionales una vía eficaz para la diversificación y distribución del riesgo hipotecario (Daher, 2013). Indiscutiblemente, la emisión de títulos respaldados con hipotecas residenciales permitió el desarrollo del sector inmobiliario estadounidense dado el reciclaje de los créditos hipotecarios destinados al financiamiento de vivienda nueva. A fines de la década de 1980, también en ese país, los valores respaldados por hipotecas subprime hicieron su debut en el mercado financiero, convirtiéndose en uno de los vehículos de mayor crecimiento del mercado de bursatilización de activos a fines de los años noventa. En 2000 y 2005, el mercado de la vivienda experimentó una rápida apreciación de los inmuebles y hubo originadores de créditos en el sector que hicieron préstamos que excedieron el valor de mercado de las casas.

Es importante notar que durante ese periodo se relajaron las calificaciones crediticias y los precios de los bienes raíces crecían constantemente, generando la expectativa de que el valor de una propiedad inmobiliaria podría superar incluso al monto de la hipoteca, como lo señala $\mathrm{Hu}$ (2011, p. 176). Sin embargo, en el 2006 los precios de los inmuebles iniciaron su declive y las alzas de las tasas de interés tuvieron como consecuencia un colapso del mercado de valores hipotecarios en 2007 dados los incumplimientos de los acreditados y el deterioro de la cartera de créditos hipotecarios otorgados, generando en 2007 y 2008 presiones recesivas en el mercado de la vivienda y la economía estadounidense, extendiéndose los efectos a la economía mundial. La desaceleración que se presentó en el sector de la vivienda en diversas economías a partir de la crisis subprime, genero perturbación financiera por el incumplimiento en el pago de hipotecas de alto riesgo en Estados Unidos, evidenciando que el sector de la vivienda puede ser un factor latente de inestabilidad macroeconómica. Es evidente, como lo señalan Reinhart y Rogoff (2011), que el sector inmobiliario se puede relacionar con crisis financieras y bancarias directamente por medio de los títulos bursatilizados con el respaldo de las carteras de créditos hipotecarios.

Para abordar este problema y contribuir a la prevención de futuras crisis financieras, es importante para los reguladores de los mercados financieros, inversionistas, emisores de activos financieros basados en hipotecas y otras 
entidades gubernamentales conocer los factores de riesgo que inciden en el deterioro las carteras que se integran por esos activos con el objetivo de bursatilizarlas. En el caso mexicano, es de señalarse que esa problemática ha sido poco estudiada. Una contribución de este trabajo es mostrar evidencia sobre variables económicas y financieras que inciden en el deterioro de la calidad de las carteras de las hipotecas bursatilizadas en México. Además, desde la perspectiva teórica, esta investigación vincula el deterioro de la calidad de esas carteras con el conocimiento que se ha acumulado por diversos estudiosos sobre el riesgo sistemático, entendido éste como el riesgo que afecta a todos los activos de un mercado o de una economía y que es un elemento fundamental en la teoría de la valuación de los activos financieros.

La siguiente sección se dedica a la revisión de literatura relevante sobre el riesgo sistemático y el deterioro de las carteras de activos basados en hipotecas. Después se describen el marco metodológico y los datos mediante los cuales se lleva a cabo el análisis que se presenta en la sección correspondiente. Finalmente se presentan las conclusiones.

\section{REVISIÓN DE LITERATURA}

En diversos trabajos de investigación se ha mostrado evidencia de que el riesgo sistemático obedece a la acción de variables económicas y financieras nacionales e internacionales; así, por ejemplo, López, (2006); He, Wang y Lai (2010); Basnet y Upadhyaya (2015); Denis y Etornam (2015); Ahmad y Ramzan (2016); Saifullah y Waqar (2017); Antonette (2018); Kahn y Kay (2019); han identificado las tasas de interés, los tipos de cambio, los precios petroleros, la inflación, PIB y desempleo, entre otras variables.

Keys, Seru y Vig (2012), investigaron la relación entre la bursatilización de hipotecas y la evaluación de los acreditados durante el periodo de la crisis subprime y señalan que la valoración poco sólida se encuentra asociada con la bursatilización en el mercado de hipotecas alto riesgo; misma que a su vez está asociada con un aumento notorio en la probabilidad de incumplimiento. Por su parte Nadauld y Sherlund (2013), considerando que las hipotecas de alto riesgo se asocian con el riesgo y tasa de incumplimiento; proporcionan evidencia empírica de que la bursatilización tuvo un impacto en las originación a través de su efecto en los incentivos de los prestamistas para disminuir el rigor de evaluación de los acreditados. Establecen que los cambios 
en las tasas de originación de préstamos con poca o ninguna documentación en la originación del crédito contribuyeron a los índices de la alta demanda de hipotecas, lo que posteriormente influyó con el incumplimiento del pago. Chee-Wooi y Brooks (2015), presentan para el mercado de valores en Malasia un estudio de los componentes del riesgo sistemático mundial y señalan que este se determina por factores macroeconómicos (riesgo político, económico y financiero) y en el riesgo financiero incluyen el PIB, inflación, tipo de cambio y la cuenta corriente de exportaciones e importaciones A partir de su estudio analizaron del riesgo sistemático en una subdivisión de la cartera mundial en cinco grupos; mercados desarrollados, regionales, mercado de EU., principales socios comerciales y el resto del mundo. Establecen que estos factores de riesgo sistemático están ligados con las crisis financieras internacionales y los regímenes económicos en Malasia en las últimas dos décadas y concluyen que dicho riesgo sistemático se determina principalmente por la fijación de precios de las empresas que cotizan en el mercado de valores y principalmente a factores estadounidenses y regionales.

Ahmad y Ramzan (2016), señalan que la tasa de interés, la oferta monetaria, el índice de precios al consumidor medido a través de la inflación, el producto interno bruto y la tasa de crecimiento de la producción industrial, son variables macroeconómicas que se encuentran interrelacionadas y la tendencia de una de éstas alteran a las otras, afectando el mercado de valores y por lo tanto a la economía de un país. A través de un modelo GARCH estudian la volatilidad de estos factores macroeconómicos en el mercado de valores de Karachi en Pakistán y muestran la dirección de la relación. Argumentan que esta relación entre el riesgo sistemático y el mercado de valores incide en la variación de los rendimientos de las acciones y específicamente los cambios en la inflación afectan el índice bursátil, en tanto que las exportaciones aumentan el flujo de efectivo, la eficiencia en el banco central y en consecuencia un incremento comercial; por otra parte, la producción industrial presenta una relación con las variaciones en dichos rendimientos y concluyen que estos factores macroeconómicos se relacionan con los rendimientos esperados en el mercado de valores.

Por otra parte, Izunobi, et al. (2017), por medio de un modelo GARCH y E-GARCG determinan que la tasa de interés, la inflación y el rendimiento del mercado para Nigeria en el periodo de 1995 a 2014 son fuente de riesgo sistemático significativo en el mercado de valores. Señalan que la relación entre el riesgo sistemático y el desempeño del mercado de valores puede 
provocar colapsos financieros, capaces de desencadenar efectos adversos en la economía. Su hallazgo revela que el riesgo sistemático es determinante en el crecimiento económico a largo plazo de un país y puede provocar efectos adversos en el portafolio de los inversionistas. En tanto que Nzomo, et al. (2017), presentan un estudio durante el periodo de 2000 a 2015 para Nigeria y Costa de Marfil y 2008 a 2015 para Camerún, de la relación de las variables económicas y financieras, como el producto interno bruto, inflación, tasa de interés, tasa de crecimiento del dinero y cuasi dinero, desempleo, índice bursátil de cierre de mercado y capitalización de mercado de empresas nacionales cotizadas, como fuente de riesgo sistemático que influye en el mercado de valores. Establecen que estas variables macroeconómicas influyen en los movimientos del mercado de valores y crecimiento económico de los países en desarrollo.

Saifullah y Waqar (2017), encuentran que los efectos de las variables macroeconómicas son fuente de riesgo sistemático en su estudio en Pakistan, donde el mercado de valores es sensible a las variaciones de estas variables y por tanto el desempeño de este afecta el progreso del país. Encuentran que en lo particular los movimientos en la tasa de interés, el tipo de cambio y la inflación pueden ejercer una influencia de mucha importancia en el mercado de valores. El modelo ARDL que utilizan en su estudio contribuye con el hallazgo de la relación de largo plazo de las variables analizadas y el índice KSE-100; mostrando que la tasa de interés tiene una influencia negativa y la inflación tiene una influencia positiva en el índice PSX a largo plazo, y las estimaciones de coeficientes de ambos regresores se encontraron altamente significativos. Asimismo, el tipo de cambio tiene un impacto positivo pero insignificante en el índice del mercado a largo plazo. El coeficiente estimado de corrección de errores del procedimiento ARDL indica que aproximadamente se corrige por año el $46.53 \%$ del desequilibrio del índice KSE-100 respecto de la ruta de su relación de equilibrio a largo plazo con las variables económicas causales del riesgo sistemático.

Lin, Yuen y Joye (2018), realizaron para el mercado de Australia un estudio en el que señalan que los precios de la vivienda y el riesgo de incumplimiento en la cartera de hipotecas bursatilizadas contribuyen con el deterioro de ésta, y señalan que las tasas de incumplimiento en las carteras de préstamos bursatilizados han aumentado considerablemente en los últimos tiempos. En tanto que las tasas de prepago presentan una fuerte disminución en el periodo de 2017 hasta finales de 2018 en dicho país. Desarrollaron un índice 
de riesgo de incumplimiento y prepago basado en una regresión hedónica a través de variables independientes que pueden incluir desde la emisión de bursatilización de la hipoteca, antigüedad promedio ponderada de los préstamos, el promedio ponderado de la relación préstamo valor de la hipoteca, que permite identificar los factores de riesgo que los prestamistas deben valorar en la calificación de los prestatarios de una hipoteca y este índice de incumplimiento hedónico incide en el deterioro.

Antonette (2018), determina la relación de los factores de riesgo sistemático y el rendimiento del mercado de valores de Sri Lanka, su estudio presenta variables macroeconómicas que influyen en dicho mercado, representadas por la tasa de interés, la inflación, oferta monetaria y el tipo de cambio e incluye el índice bursátil en el periodo 1998 a 2016. Estima un VECM y un modelo EGARCH y determina una relación de largo plazo entre las variables macroeconómicas y los rendimientos del mercado mediante metodología de análisis de la cointegración propuesta por Johansen y Juselius. Encuentra que la oferta monetaria y la tasa de interés son causales de riesgo sistemático, concluyendo así que estos hallazgos contribuyen en la formulación de políticas económicas y financieras, en tanto que inversionistas y analistas de mercado deben observar el riesgo sistemático que revelan estas variables, para su estrategia de diversificación y estructura de portafolio. En este sentido, Karki (2018), examina factores macroeconómicos como fuente de riesgo sistemático para el mercado de valores de Nepal, considerando la inflación, tasa de interés, producto interno bruto (PIB) y la oferta monetaria, en el periodo de estudio de 1994 a 2016. Su estudio revela que ese mercado de valores responde positivamente al PIB, la inflación y la oferta monetaria y negativamente a la tasa de interés.

Kahn y Kay (2019), proporcionan evidencia de cómo los cambios en la oferta de crédito incidieron en el auge y caída de las hipotecas de alto riesgo en los Estados Unidos. Enuncian que el auge de las hipotecas de alto riesgo tiene origen en la fijación de precios erróneos del riesgo, así como en el optimismo de los precios de la vivienda. El precio erróneo deriva en gran medida de la agrupación de riesgos crediticios muy disímiles y dio un resultado adverso dentro del grupo. Así, los precios de la vivienda (Mian y Sufi, 2009) y el optimismo (Adelino et al., 2016), para diferentes segmentos del mercado, evidencian el riesgo de incumplimiento.

Jedidi y Dionne (2019), sostienen que la decisión de cambio de servicios en la bursatilización de hipotecas puede afectar el incumplimiento del 
préstamo y muestran que la probabilidad de cambio del administrador hipotecario incrementa la probabilidad de que el prestatario no cumpla. Su investigación sugiere que el originador del préstamo utilizando información privilegiada sobre las características de riesgo del préstamo y la calidad crediticia del prestatario, realiza la bursatilización de las hipotecas para transferir el riesgo de incumplimiento; así, el originador podría retener préstamos de calidad superior con una baja probabilidad de incumplimiento en su cartera de servicios y vender los créditos con alto riesgo de incumplimiento.

Morgan, et al. (2019), presentan un estudio en el que establecen que las políticas macroprudenciales contribuyen con la regulación y supervisión del riesgo sistemático en los mercados inmobiliarios; para su estudio incluyeron información de 4000 bancos de 46 países y analizaron la relación préstamo-valor y la política macroprudencial en el otorgamiento de préstamos hipotecarios en países en desarrollo para la predicción de crisis bancarias globales. es De acuerdo con ellos, las políticas macroprudenciales ayudan a detectar y atenuar los riesgos que perturban la estabilidad sistémica, teniendo como objetivo reducir el costo económico que involucra esta perturbación de los mercados financieros. Para su estudio manejan variables bancarias (valor de la vivienda, préstamos deteriorados) y económicas (tasa de interés del préstamo, PIB, índice de precios de la vivienda). Además, esos autores destacan la importancia del monitoreo de las variables globales que permitan evaluar los riesgos para la estabilidad financiera y variables específicas del país como determinantes de los préstamos hipotecarios.

Longstaff y Rajan (2008) presentaron evidencias de que el riesgo de crédito en toda la economía ha ido en aumento de forma espectacular después de 2007 y fue el factor principal del aumento del incumplimiento durante el 2007, deduciendo por tanto que los problemas de crédito macroeconómicos han generado un gran impacto en el riesgo de crédito total; en tanto que Das et al. (2007) señalan que a partir de indicadores macroeconómicos como el Producto Interno Bruto y la tasa de crecimiento de la producción industrial es posible representar el riesgo sistemático. Ante esta incertidumbre de incumplimiento en el pago del crédito de hipotecas, en países como Estados Unidos, España, Islandia, e Irlanda de manera precisa, se han orientado políticas y sistemas al rescate bancario ante los estragos financieros; estos mecanismos aplicados en las instituciones de crédito buscan incrementar procedimientos y técnicas para la medición de los riesgos de los créditos y contrarrestar situaciones y eventos relacionados con dicho incumplimiento. 
Landreth y Colander (2006), reconocen que un elemento del incumplimiento del crédito es la incertidumbre en los mercados financieros, mientras que la innovación financiera de bursatilización de activos respaldados con hipotecas ha generado tanto la diversificación de beneficios como crisis financieras que han originado grandes niveles de deterioro en la economía de diversos países (La Caixa, 2008). Así, por ejemplo, la turbulencia financiera en Asia comenzó con la exposición desfavorable del riesgo para préstamos de bienes raíces de Tailandia, y también el temor de la economía de Tailandia produjo un efecto dominó en los países vecinos, incluyendo Singapur, Malasia, Indonesia, Hong Kong, Taiwán y Corea del Sur (Liu y Mei, 2003).

Fisher (1930), mostró con evidencia empírica que la tasa de interés responde a variaciones de la inflación, en el sentido y magnitud predichos por la teoría. De este modo, las variaciones en la tasa de interés de acuerdo con Merton (1973) son cambios que capturan los cambios en el conjunto de oportunidades de inversión. De acuerdo con Irvin Fisher (1990), la tasa de interés incide directamente en la inversión y, por lo tanto, cuando se tienen altas tasas de interés un proyecto ya no es atractivo ni rentable. La tasa de interés constituye una variable relevante por su dependencia directa con respecto al crédito (Maza y González, 1992). Cuando la tasa de interés varía, el nivel de precios varía en la misma proporción y sentido, por lo que la tasa de interés en el mediano y largo plazo tiene variaciones marginales en el corto plazo.

El desempleo, como lo establecieron estudios realizados por Brüggeman (2006), Mayes y Vilmunen (1999), Kitov, Kitov y Dolinskaya (2007), es una variable que tiene correspondencia en una relación de largo plazo entre los salarios reales; y de forma particular es una composición de choques en la productividad, demanda y oferta laboral, y que determina su comportamiento. Por su parte, los trabajos de Zenteno (2002) y Rojas (2004), mostraron cómo el desempleo, en época de dificultades económicas severas como en la crisis de 1995 en México, es una variable que está representada como un fenómeno generalizado en las diversas áreas del país, y que la magnitud y sentido de dicho incremento tiene relación con la especialización económica local.

El comportamiento de la inflación tiene consecuencias sobre el crecimiento económico. Por su parte Mundell (1963), estableció que la inflación induce a una mayor expansión de la producción, debido a que indirectamente aumenta los niveles de ahorro de la economía y el acervo de capital, y reduce de forma inmediata la riqueza de las personas, por lo que estas se ven obligadas a incrementar su ahorro a fin de recuperar los niveles de 
riqueza previos. En este sentido, Mendoza (1998) concluyó que en el largo plazo la inflación prácticamente no tiene efecto alguno sobre el crecimiento, y las referencias que existen sobre este tema para el caso de México adoptan un enfoque lineal, por lo que sus resultados identifican una relación negativa entre la inflación y el crecimiento de pequeña magnitud.

Las variaciones en el tipo de cambio representan una variable que impacta en la percepción de los ingresos de las familias, la cual puede explicar y medir estadísticamente las alteraciones en los precios de las viviendas (Maddala y Kim, 1998). Para la economía de los países en vías de desarrollo y países desarrollados, las variaciones del tipo de cambio han sido siempre un tema central con respecto en la política económica (Edwards, 1989). En México, y considerando su economía en desarrollo, la variable tipo de cambio tiene importancia y relevancia por la captación de las relaciones internacionales, y por lo tanto es una variable determinante para la estabilidad de la economía.

En México, durante el año 1994 la apreciación real del peso frente al dólar fue justificada por movimientos en los fundamentales (diferenciales de productividad, apertura del país al comercio internacional, tarifas de importación y gasto del gobierno). Además, que estas variaciones en el tipo de cambio tienen relación con la diferencia de precios entre México y Estados Unidos (Hegwood y Papell, 1998). En términos de política económica el comportamiento del mercado cambiario causa el comportamiento de los precios relativos. El riesgo de las variaciones o fluctuación en la moneda peso-dólar en actividades de inversión o en instrumentos o activos financieros establece un riesgo que perturba directamente los activos y pasivos del inversionista, lo cual origina desviaciones en el margen de sus utilidades.

Rosen (1974), en su teoría de precios hedónicos, explicó que el precio de bienes heterogéneos (viviendas) representa el valor asignado por el mercado a un bien raíz, mediante lo cual es posible determinar cómo cambiará dicho valor al variar la cantidad y calidad en que se encuentra presente cada uno de estos atributos (superficie, aptitud de uso del suelo, calidad de la construcción, diseño interior y exterior, áreas verdes, ubicación, características del vecindario), y consecuentemente, predecir precios; es decir, los bienes son valuados por la utilidad que brindan sus atributos o características. A su vez, Laeven y Valencia (2008), Roubini y Mihm (2010), Reinhart y Rogoff (2011) establecieron que el ciclo de los precios de la vivienda en el sector inmobiliario se relaciona con las crisis bancarias y financieras. La política monetaria incide en los precios de la vivienda, derivado de los efectos de las tasas 
de interés y de las burbujas de precios de los activos; siendo este un factor fundamental en el control del riesgo sistemático en el mercado de valores.

Lardic y Mignon (2008); Jiménez-Rodríguez y Sánchez (2005), afirmaron que los cambios en el precio del petróleo inciden en los mecanismos de oferta y demanda de la actividad económica, en las inversiones en los mercados financieros, así como también tienen impactos en el consumo, el gasto en los hogares y en tanto los precios presenten tendencia alta, puede forzar a los hogares a recortar sus gastos en otros bienes y servicios. He, Wang y Lai (2010), Basnet y Upadhyaya (2015) y Denis y Etornam (2015), establecen que los precios del petróleo determinan el comportamiento económico; por su parte, Zaidi, Karim y Azman-Saini (2013), plantearon que el vínculo entre choques extranjeros del precio del petróleo y las variables macroeconómicas es relevante y trascendente en la determinación de políticas para la estabilidad económica en los países.

\section{Sobre la metodología y los datos}

El modelo ARDL (Autoregresivo de rezagos distribuidos), desarrollado por Pesaran, Shin y Smith (2001), es un modelo dinámico entre cuyas ventajas cuenta que también permite comprobar la presencia de una relación cointegrante o de largo plazo entre las variables que se encuentran bajo análisis (Banerjee et al. 1993). Una ventaja importante es que permite detectar la existencia de cointegración incluso en el caso de que la especificación del modelo incluya una combinación de variables $I(0)$ e $I(1)$.

El modelo ARDL se puede especificar en principio como:

$$
y_{t}=a_{0}+a_{1} t+\sum_{i=1}^{p} f i y_{t-i}+\sum_{j=0}^{p} \beta_{j}^{\prime} \vec{x}_{t-j}+u_{t}=\max (p . q), \ldots, T
$$

Para someter a prueba la hipótesis nula de no cointegración, Pesaran, Shin y Smith (2001) proponen una reparametrización, de tal manera que el modelo incluye un mecanismo de corrección de errores:

$$
\Delta y_{t}=\alpha_{0}+\alpha_{1} t+\varsigma\left(y_{t-1}-\vec{\theta} \vec{x}_{t-1}\right)+\sum_{i=1}^{p-1} \psi_{i} \Delta y_{t-i}+\vec{\omega}^{\prime} \Delta \vec{x}_{t}+\sum_{j=1}^{q-1} \vec{\varphi}^{\prime} \Delta \vec{x}_{t-j}+\xi_{t}
$$

determinándose el coeficiente de la velocidad del ajuste por $\varsigma=1-\sum_{i=1}^{p} \phi_{i}$, en tanto que los coeficientes de largo plazo están determinados por $\theta_{k j}=\frac{\sum_{j=0}^{q} \beta_{k j}}{\varsigma}$

La hipótesis nula de no relación de largo plazo, o no cointegración, entre los niveles de las series se plantea como la prueba ligada condicional: 


$$
H_{0}:(\alpha=0) \cap\left(\sum_{j=0}^{q} \beta_{j}=0\right)
$$

El valor calculado para el estadístico $F$ derivado de esta prueba se compara con los valores críticos proporcionados por Pesaran et al. (2001).

Las observaciones mensuales, de diciembre de 2008 a junio de 2016, sobre el índice de deterioro de la calidad de las carteras hipotecarias bursatilizadas en México, se obtuvieron de la página web de la Comisión Nacional Bancaria y de Valores. Es conveniente destacar que la información estadística del Índice de Deterioro de títulos respaldados con hipotecas sólo se publicó por la CNBV para dicho periodo, por lo que el análisis cuyos resultados se muestran en esta sección se restringe por necesidad a él.

Las variables consideradas como causas de riesgo sistemático son el Índice de Precios de la Vivienda (VIV), tasa de rendimiento de los Certificados de la Tesorería de la Federación a 28 días (CET), Índice Nacional de Precios al Consumidor (INPC), tasa de desocupación de la Encuesta Nacional de Ocupación y Empleo (DES), base monetaria (BASE), Indicador Global de la Actividad Económica (IGAE), tipo de cambio pesos nacionales por dólar norteamericano (TC), precio promedio de exportación de petróleo (PET), Índice de Precios y Cotizaciones de la Bolsa Mexicana de Valores (IPC), reservas internacionales (RI), balanza comercial de mercancías (BAL), tasa de rendimiento promedio de la Letra de la Tesorería de Estados Unidos a un mes (IEU). La muestra de todas las variables, incluyendo el deterioro, tiene periodicidad mensual y comprenden el periodo de estudio de diciembre de 2008 a junio de 2016. Las fuentes de las series económica son Banxico e Inegi.

Además de esas variables, se proponen adicionalmente para este trabajo de investigación otras variables que pueden considerarse como fuentes de riesgo sistemático en nuestro país. En primer lugar se incluye la Balanza Comercial de Mercancías de México (BCMM), como un posible factor de riesgo sistemático que permite identificar los posibles efectos en la situación económica de México en relación con sus actividades de comercio internacional, López (2006) encontró evidencia de la relevancia de las actividades de comercio con el exterior, exportaciones e importaciones, en este documento nos concentramos en el saldo de esa cuenta la cual indica la presencia de un déficit o superávit en el comercio con el exterior.

Por su parte, la Base Monetaria, también denominada dinero de alta potencia, se puede considerar como posible factor de riesgo sistemático debido 
a los efectos que pudiera tener en la variación de los niveles de liquidez en la economía, los cuales pueden afectar a diversas variables económicas importantes como lo aon el nivel general de la actividad económica, la expansión del crédito interno, inversión en los mercados financieros, pudiendo tener efectos en el nivel general de los precios. De manera obvia, la dinámica económica puede ser también una variable de riesgo sistemático en la medida en la cual puede afectar el comportamiento de otras variables y, en última instancia, a los distintos agentes económicos. Para representar la actividad económica en México se seleccionado el Indicador Global de la Actividad Económica (IGAE) pues se trata de un indicador ampliamente conocido y empleado para considerar el desempeño de la economía.

\section{Análisis del deterioro en la cartera de hipotecas bursatilizadas}

Como puede observarse en el panel inferior del cuadro 1, la prueba ligada de Pesaran et al. (2001) rechaza la hipótesis ligada condicional de no cointegración, es decir, se confirma la existencia de una relación de largo plazo entre las variables analizadas (deterioro y el conjunto de variables).

En el panel superior del cuadro 1 se muestran también los resultados de la parametrización como mecanismo de corrección del error del modelo autoregresivo con rezagos distribuidos estimado. Para el logaritmo del índice de deterioro las elasticidades de corto plazo de $\triangle I G A L_{t-1}(8.20 \mathrm{E}-13)$, $\triangle B A S E_{t-1}(0.447954), \triangle B A S E_{t-2}(0.570396), \triangle I E U(408.2453), \Delta I G A E_{t-1}(0.867907)$, $\Delta I N P C_{t-1}(-4.179938), \Delta I P C_{t-1}(-0.295495), \Delta T C(-0.620157), \Delta V I V_{t-2}(-20.62069)$ son estadísticamente significativas al 1\%. De esta forma, se determina una relación positiva entre la dinámica de los cambios de deterioro y balanza comercial rezagada en un mes, base monetaria con uno y dos rezagos, tasa de rendimiento de letras del tesoro con un rezago, índice general de actividad económica con un rezago, índice nacional de precios al consumidor, y precios de vivienda con dos rezagos; en tanto que también se encontró evidencia de una relación negativa entre la dinámica de los cambios de deterioro y el índice de precios y cotizaciones con un rezago y tipo de cambio en un periodo.

Por otra parte, los coeficientes de $\triangle B A L(-4.92 \mathrm{E}-13), \triangle I N P C_{t-2}(-2.404242)$, son estadísticamente significativas al $5 \%$. Por lo que, de acuerdo con el estudio respecto a la dinámica de los cambios en la balanza comercial con un rezago e índice nacional de precios y cotizaciones con dos rezagos, presentan una consecuencia negativa en los cambios de deterioro. 
Así mismo, los coeficientes de $\triangle I N P C_{t-1}$ (2.159375), $\triangle I P C(0.158251)$, $\Delta I P C_{t-2}(-0.12494)$ son estadísticamente significativas al $10 \%$. Es así como

\section{Cuadro 1}

Modelo ARDL estimado como mecanismo de corrección del error

\begin{tabular}{|c|c|c|c|}
\hline \multicolumn{4}{|c|}{$\begin{array}{l}\text { Variable dependiente: cambios en el índice de deterioro de la calidad } \\
\text { de las carteras hipotecarias bursatilizadas en México }\end{array}$} \\
\hline Variable & Coeficiente & Error estándar & Estadístico $t$ \\
\hline$\triangle B A L$ & $-4.92 \mathrm{E}-13$ & $1.97 \mathrm{E}-13$ & $-2.50179 * *$ \\
\hline$\triangle B A L_{t-1}$ & $8.20 \mathrm{E}-13$ & 2.19E-13 & $3.737893^{* * *}$ \\
\hline$\triangle B A S E$ & 0.055949 & 0.087609 & 0.638622 \\
\hline$\triangle B A S E_{t-1}$ & 0.447954 & 0.116543 & $3.843684^{* * *}$ \\
\hline$\triangle B A S E_{t-2}$ & 0.570396 & 0.12146 & $4.696146^{* * *}$ \\
\hline$\triangle I E U$ & 408.2453 & 109.5609 & $3.726197^{* * *}$ \\
\hline$\triangle I G A E$ & 0.125534 & 0.123069 & 1.020036 \\
\hline$\triangle I G A E_{t-1}$ & 0.867907 & 0.1243 & $6.982369^{* * *}$ \\
\hline$\triangle I N P C$ & -4.179938 & 1.029936 & $-4.058444^{* * *}$ \\
\hline$\triangle I N P C_{t-1}$ & 2.159375 & 1.189273 & $1.815711^{*}$ \\
\hline$\triangle I N P C_{t-2}$ & -2.404242 & 0.985619 & $-2.439321^{* *}$ \\
\hline$\triangle I P C$ & 0.158251 & 0.0827 & $1.913543^{*}$ \\
\hline$\Delta I P C_{t-1}$ & -0.295495 & 0.0894 & $-3.305295^{* * *}$ \\
\hline$\Delta I P C_{t-2}$ & -0.12494 & 0.074692 & $-1.672747^{*}$ \\
\hline$\triangle T C$ & -0.620157 & 0.146116 & $-4.244278^{* * *}$ \\
\hline$\triangle V I V$ & 2.052566 & 6.242642 & 0.328798 \\
\hline$\Delta V I V_{t-1}$ & -2.614798 & 7.507346 & -0.348299 \\
\hline$\Delta V I V_{t-2}$ & -20.62069 & 5.521539 & $-3.734591^{* * *}$ \\
\hline$T C E 1_{t-1}$ & -0.327259 & 0.037896 & $-8.635741^{* * *}$ \\
\hline \multicolumn{4}{|c|}{${ }^{* * *},{ }^{* *} \mathrm{y}^{*}$ denotan significancia al 1\%,5\% y $10 \%$, respectivamente. } \\
\hline \multicolumn{4}{|c|}{ Prueba de cointegración de Pesaran y Shin } \\
\hline Estadístico $F$ & $\begin{array}{c}\text { Nivel de } \\
\text { significancia }\end{array}$ & \multicolumn{2}{|c|}{$\begin{array}{l}\text { Valores críticos } \\
I(0) \quad I(1)\end{array}$} \\
\hline \multirow[t]{4}{*}{4.323} & $10 \%$ & 1.76 & 2.8 \\
\hline & $5 \%$ & 1.98 & 3 \\
\hline & $2.50 \%$ & 2.18 & 3.3 \\
\hline & $1 \%$ & 2.41 & 3.6 \\
\hline
\end{tabular}

Fuente: elaborción propia. 
se encontró que, los cambios en el índice nacional de precios al consumidor con un rezago y el índice de precios y cotizaciones con rezago en un mes, se observa que presentan un efecto positivo, pues el índice de precios y cotizaciones con un rezago, tienen un efecto negativo en los cambios de deterioro. Por último, el coeficiente de TCE1 ${ }_{t-1}(-0.327259)$ es estadísticamente significativo al 10\%. De esta manera, la velocidad de convergencia entre el corto y largo plazo del índice de deterioro estimada muestra que las variables no están en equilibrio, sin embargo, este equilibrio se restablece en un mes alrededor del $32.72 \%$.

De esta forma, es posible establecer, que el tipo de comercio que el país realiza ejerce influencia clave en los posibles riesgos que inciden en el deterioro de la cartera de hipotecas bursatilizadas. Así mismo, se encontró evidencia de la influencia que ejercen los cambios de la variabilidad en el ajuste de la base monetaria que efectúa el Banco de México en la economía, de un posible riesgo que aumenta el deterioro, es decir, los cambios que se producen entre la demanda de dinero, al inyectar o quitar liquidez como parte de la política monetaria, muestran una evidencia de un aumento que contribuye razonablemente en las variables que la explican el deterioro de dicha cartera.

En tanto que la variabilidad en la tasa de rendimiento promedio de la Letra de la Tesorería de Estados Unidos a un mes, en relación con la evidencia que se encontró en este trabajo de investigación como posible factor de riesgo establece que los choques en dicha variable y la relación de largo, producen un aumento el deterioro de la cartera de hipotecas bursatilizadas.

Por otra parte, la evidencia del estudio de la relación en la variabilidad que muestra el Indicador Global de la Actividad Económica con el deterioro de la cartera de hipotecas bursatilizadas, en el periodo de estudio, establece un posible factor de riesgo que actúa en respuesta a la información que revela el comportamiento real de la economía. También se observa que el Índice Nacional de Precios del Consumidor, es también un posible factor de riesgo que contribuye a la explicación de la variabilidad en el deterioro de la cartera de hipotecas bursatilizadas en México, es decir, los cambios en el poder adquisitivo en los acreedores hipotecarios, en un ambiente de inflación alta, conducen al deterioro, toda vez que se enfrenta incertidumbre acerca del comportamiento de los precios en la economía, así, el poder de compra, se ve deteriorado directamente por la inflación y por lo tanto, existe una correlación entre los préstamos individuales o un grupo de préstamos 
hipotecarios, es decir, las condiciones económicas en el país y las condiciones del mercado, son un factor de percepción del riesgo para los inversionistas. En este sentido, el efecto de la inflación en el mercado de hipotecas en condiciones vulnerables produce un aumento al deterioro de dicha cartera.

Por otra parte, el Índice de Precios y Cotizaciones de la Bolsa Mexicana de Valores muestra evidencia en la variabilidad del aumento del deterioro en la cartera de hipotecas bursatilizadas, se encontró en esta variable un posible factor de riesgo que produce aumentos por la supuesta disociación que existe entre las utilidades que proceden del diferencial de precios de las acciones en el mercado bursátil de la Bolsa Mexicana de Valores (BMV) y el comportamiento real de la economía. Así mismo, se determinó que el tipo de cambio pesos nacionales por dólar norteamericano, produce un aumento significativo en el deterioro de la cartera de hipotecas bursatilizadas, la variabilidad de este posible factor de riesgo puede estar determinado porque tiene efectos concluyentes sobre el comportamiento de la economía, es decir, el comportamiento del mercado de divisas ejerce fuerzas en la oferta y demanda de divisas y por lo tanto determina el valor de cada divisa extranjera.

\section{Conclusiones}

En este trabajo se han presentado los resultados del estudio de la relación entre el deterioro de la calidad de la cartera de hipotecas bursatilizadas en México y variables económicas y financieras, tanto internas como internacionales, que la literatura ha identificado como causales del riesgo que enfrentan los activos de una economía. Por medio de un modelo autoregresivo de rezagos distribuidos, se encontró evidencia de posibles factores de riesgo que tienen efectos significativos en la variabilidad del comportamiento del deterioro de la cartera de hipotecas bursatilizadas para el período de estudio de diciembre de 2008 a junio de 2016.

La evidencia muestra que la balanza comercial de mercancías, base monetaria, tasa de rendimiento promedio de la Letra de la Tesorería de Estados Unidos a un mes, Indicador Global de la Actividad Económica, Índice Nacional de Precios al Consumidor, Índice de Precios y Cotizaciones de la Bolsa Mexicana de Valores, tipo de cambio pesos nacionales por dólar norteamericano y el Índice de Precios de la Vivienda, tienen una relación 
de equilibrio de largo plazo con el deterioro. La evidencia mostrada en este trabajo es congruente con los hallazgos de otros trabajos de investigación como el de López (2006), Keys et al. (2012); Chee-Wooi y Brooks (2015); Ahmad y Ramzan (2016); Izunobi, et al. (2017); Nzomo, et al. (2017); Saifullah y Waqar (2017); Lin et al. (2018); Antonette (2018); Karki (2018); Kahn y Kay (2019); Jedidi y Dionne (2019) y Morgan, et al. (2019).

El Índice de Precios de la Vivienda, produce una disminución en el deterioro de la cartera de hipotecas bursatilizadas, es decir un incremento de los precios en la vivienda, como posible factor de riesgo, puede representar un incentivo para el acreditado por la revaluación de su patrimonio, en este sentido, este posible factor de riesgo contribuye a disminuir el incumplimiento de los pagos en las hipotecas y por tanto en el deterioro de dicha cartera. Nuestros resultados son consistentes con los trabajos de Jianping Mei y Hsien-Hsing Liao (2003), Laeven y Valencia (2008), Roubini y Mihm (2010), Reinhart y Rogoff (2011).

Como nos han mostrado los hechos, el mercado de las hipotecas bursatilizadas puede generar una dinámica capaz de producir fuertes desequilibrios financieros y económicos, por lo que, para el mercado de activos financieros, inversionistas y autoridades regulatorias, es importante comprender el proceso de incumplimiento en las hipotecas, en particular las causas que pueden llevar al deterioro de las carteras crediticias. Se debe reconocer que sin lugar a duda los otorgantes de créditos para hipotecas, como son los bancos y otros organismos especializados, pueden conocer de primera mano y con lujo de detalle cuáles son las variables relevantes para calificar a los potenciales acreditados. Así mismo pueden contar también con los elementos técnicos suficientes para cuantificar el impacto de variables microeconómicas en el incumplimiento por parte de quienes gozan de los créditos concedidos. Sin embargo, en ese sentido, siendo uno de los primeros en abordar esa problemática, este trabajo contribuye a la agenda de investigación necesaria para avanzar en el conocimiento sobre las causas del incumplimiento desde la perspectiva de los acreditados, en particular cuando los créditos hipotecarios concedidos a través de los canales convencionales se empaquetan y se comercializan en el mercado bursátil, caso en el cual el inversionista y otros participantes del mercado desconocen los pormenores del contenido de las carteras bursatilizadas.

Queda demostrado en estas páginas, que la literatura del riesgo sistemático proveniente de fuentes macroeconómicas puede proporcionar un marco 
de referencia importante para entender el deterioro de la calidad de las carteras hipotecarias bursatilizadas en México, es decir, el alcance de la teoría sobre los factores de riesgo sistemático podría permitir valuar no sólo activos de capital sino también activos financieros emitidos con base en créditos hipotecarios. Posiblemente esa contribución de la teoría de valuación de activos de capital pueda complementar la teoría que los estudiosos del tema han desarrollado para explicar el incumplimiento de los acreditados con base en planteamientos del nivel microeconómico.

\section{Referencias}

Adelino, Manuel, Schoar Antoinette, Felipe Severino (2016). Loan Originations and Defaults in the Mortgage Crisis: The Role of the Middle Class, The Review of Financial Studies, 29(7), 1635-1670. DOI: 10.1093/rfs/hhw018.

Ahmad, N., M. Ramzan (2016). Stock Market Volatility and Macroeconomic Factor Volatility. International Journal of Research in Business Studies and Management. $3(7), 37-44$.

Antonette, F. (2018). Macroeconomic Impact on Stock Market Returns and Volatility: Evidence from Sri Lanka. Business and Economics Journal, 9, 379. DOI: 10.4172/2151-6219.1000379.

Basnet, H., \& K. Upadhyaya (2015). Impact of oil price shocks on output, inflation and the real exchange rate: evidence from selected ASEAN countries. Applied Economics. 47, 3078-3091.

Bullard, J.; C. Neely \& D. Wheelock (2009). Systemic risk and the financial crisis: $a$ primer. Review, Federal Reserve Bank of St. Louis, 91(5), 403-418.

Brüggemann, R. (2006). Sources of German Unemployment: A Structural Vector Error Correction Analysis. Empirical Economics, 31 (2), 409-431. 
Chee-Wooi, H. \& R. D. Brooks (2015). The components of systematic risk and their determinants in the Malaysian equity market. 11. 151-176.

Daher, A. (2013). El sector inmobiliario y las crisis económicas. Revista EURE. 39 (118), 47-76.

Daher, A. (2013). Fondos inmobiliarios y riesgo urbano. Revista de Urbanismo. (29), $32-45$.

Das, S.; D. Duffie; N. Kapadia \& L. Saita (2007). Common failings: how corporate defaults are correlated. The Journal of Finance. LXII (1), 93-118.

He, Y.; S. Wang \& K. Lai (2010). Global economic activity and crude oil prices: A cointegration analysis. Energy Economics. 32(4), 868-876.

Denis, D., y D. K. Etornam (2015). Granger causality analysis on Ghana's macroeconomic performance and oil price fluctuations. Journal of Resources Development and Management, 6, 1-5.

Edwards, S. (1989). Exchange rate misalignment in developing countries. World Bank Research Observer, 4(1), 3-21.

Fisher, I. (1930). The Theory of Interest. New York: Macmillan Publishers Ltd.

Fisher, R. (1990). The social psychology of intergroup and international conflict resolution. Nueva York: Springer-Verlag.

Gujarati, D., \& D. Porter (2010). Econometría, 5a edición. México, D.F.: McGrawHill.He.

Y. Wang, S. \& K. Lai (2010). Global economic activity and crude oil prices: A cointegration analysis. Energy Economics, 32(4), 868-876.

Hegwood, N. \& D. Papell (1998). Quasi Purchasing Power Parity. International Journal of Finance and Economics, 3(4), 279-289.

Hooy, Chee-Wooi \& D. Brooks, Robert (2015). The Components of Systematic Risk and Their Determinants in The Malaysian Equity Market, Asian Academy of Management Journal of Accounting and Finance (AAMJAF), 11(2), 151-176.

$\mathrm{Hu}$, J. (2011). Asset Securitization: Theory and Practice. Singapore: John Wiley \& Sons. Izunobi Anthony Okechukwu, et. al (2017). Macroeconomic Variables Volatility in the Nigerian Stock Market; An Empirical Analysis. International Journal of Managerial Studies and Research,5(6),1-13. DOI:10.20431/2349-0349.0506001.

Jedidi, Helmi \& Dionne, Georges, Testing for Information Asymmetry in the Mortgage Servicing Market (March 12, 2019). DOI:10.2139/ssrn.3351417.

Jianping, M. \& L. Hsien-Hsing (2003). Asset Pricing. Singapur: World Scientific Publishing.Jiménez-Rodríguez, R., y M. Sánchez (2005). Oil price shocks and real GDP growth: empirical evidence for some OECD countries. Applied Economics, 37(2), 201-228. 
Kahn, James A. \& S. Kay, Benjamin (2019). The Impact of Credit Risk Mispricing on Mortgage Lending during the Subprime Boom. Finance and Economics Discussion Series 2019-046, Board of Governors of the Federal Reserve System (US). DOI: 10.17016/FEDS.2019.046.

Karki, D. (2018). Stock Market Responses to Macroeconomic Dynamics: Testing for Long-Run Equilibrium in Nepal. Pravaha, 24(1), 64-82. DOI: 10.3126/pravaha. v24i1.20227.

Kitov, I., O. Kitov \& S. Dolinskaya (2007). Inflation as a function of labor force change rate: cointegration test for the USA. MPRA Paper 2734. München: University Library of Munich.

Keys, Benjamin J., Seru, Amit, Vig, Vikrant (2012). Lender Screening and the Role of Securitization: Evidence from Prime and Subprime Mortgage Markets, The Review of Financial Studies, 25(7), 2071-2108. DOI: 10.1093/rfs/hhs059.

La Caixa. (2008). Convulsión en el sistema financiero. Informe Mensual, 317. Barcelona: Servicio de Estudios de La Caixa.

Laeven, Luc and Fabian Valencia (2008). "Systemic Banking Crises: A New Database," IMF Working Paper No. 08/224.

Landreth, H., \& D. Colander (2006). Historia del pensamiento Económico. México, D.F.: Editorial CECSA.

Lardic, S., y V. Mignon (2008). Oil prices and economic activity: An asymmetric cointegration approach. Energy Economics, 30(3), 847-855.

Lin, Kai \& Yuen, Ainslie \& Joye, Christopher (2018). A New Hedonic Method for Measuring Mortgage Default Risk and Prepayment Rate (October 15, 2018). DOI: $10.2139 /$ ssrn.3237169.

Liu, C., \& J. Mei (2003a). A Time-varying Risk Analysis of Equity and Real Estate Markets in the U.S. and Japan. Asset Pricing. 1, 77-99.

Longstaff, F., \& A. Rajan (2008). An Empirical Analysis of the Pricing of Collateralized Debt Obligations. Journal of Finance. (63), 529-563.

López, F. (2006). Riesgo sistemático en el mercado mexicano de capitales: un caso de segmentación parcial. Contaduría y Administración. (219), 86-113.

Maddala, G., \& K. In-Moo (1998). Unit Roots, Cointegration, and Structural Change. Cambridge: Cambridge University Press.

Mayes, D., \& V. Jouko (1999). Unemployment in a Small Open Economy: Finland and New Zealand. Bank of Finland Discussion Papers, No. 10. Helsinki: Bank of Finland.

Maza, D., \& A. González, (1992). Tratado moderno de economía. Caracas: ed. Panapo. 
Mendoza, M. A. (1998). Inflación y crecimiento económico en México. Monetaria. Revista del CEMLA, 21(2).

Merton, R. (1973a). An Intertemporal Capital Asset Pricing Model. Econometrica, 41 (5), 867-887.

Merton, R. (1973b). The theory of rational option pricing. Bell Journal of Economics and Management Science, 4 (1), 141-183.

Mian,A \& A. Sufi (2009). The Consequences of Mortgage Credit Expansion: Evidence from the US. Mortgage Default Crisis. The Quarterly Journal of Economics, 124 (4), 1449-1496. DOI:10.1162/qjec.2009.124.4.1449.

Morgan, Peter J.; J. Regis Paulo, Salike, Nimesh (2019). LTV policy as a macroprudential tool and its effects on residential mortgage loans. Journal of Financial Intermediation, 37, 89-103. DOI:10.1016/j.jfi.2018.10.001.

Mundell, R. (1963). Inflation and Real Interest. Journal of Political Economy, 71.

Nadauld, Taylor D. \& M. Sherlund, Shane (2013). The impact of securitization on the expansion of subprime credit. Journal of Financial Economics, Elsevier, 107(2), 454-476. DOI: 10.1016/j.jfineco.2012.09.002.

Nzomo T, Joseph \& Dombou-Tagne, R. Dany (2017). Stock markets, volatility and economic growth: evidence from Cameroon, Ivory Coast and Nigeri. Panorama Económico, Escuela Superior de Economía, Instituto Politécnico Nacional, vol. 12(24), 145-175.

Reinhart, C. and K. Rogoff (2009). This Time is Different: Eight Centuries of Financial Folly, Princeton University Press.

Reinhart, C. and K. Rogoff (2011). Testa vez es distinto: ocho siglos de necedad financiera. México, DF. Fondo de Cultura Económica.

Rojas García, Georgina (2004). Precariedad laboral en el México urbano de fines del siglo xx: comparación de 38 mercados locales de trabajo, en Fernando Lozano Ascencio (coord.), El amanecer del siglo y la población mexicana, Cuernavaca, Centro Regional de Investigaciones Multidisciplinarias, Universidad Nacional Autónoma de México/Sociedad Mexicana de Demografía, pp. 553-572.

Rosen, S. (1974). Hedonic Prices and Implicit Markets: Product Differentiation in Pure Competition. Journal of Political Economy, 82(1), 34-55.

Roubini, N., \& Nihm, S. (2010). Crisis economics, a crash course in the future of finance. New York; Penguin Press.

Saifullah, Khan \& Waqar Khalid (2017). Effects of Macroeconomic Variables on the Stock Market Volatility: The Pakistan Experience. International Journal of Econometrics and Financial Management, 5 (2), 42-59. DOI: 10.12691/ijefm-5-2-4.

Suárez, R. (2006). La bursatilización de activos en México y su aplicación en el sector carretero del país [Tesis doctoral]. México, DF.: ITESM. 
Margarita M. Mosso Martínez, Francisco LóPeZ-Herrera

Zaidi, M. A. S.; Z. A. Karim y W. N. W. Azman-Saini (2013). Foreign and domestic shocks: Macroeconomic responses of ASEAN-3 Countries. Global Economic Review, 42(3), 215-237.

Zenteno, René (2002). Tendencias y perspectivas en los mercados de trabajo local en México: ¿más de lo mismo? Población y sociedad al inicio del siglo XXI, El Colegio de México, México. 